\title{
A Comparative Study on the Interference Microscopy Imaging and Diffraction Microscopy Imaging for Biological Cells
}

\author{
Minjie Liang, ${ }^{1, \mathrm{a}}$, Ying Ji ${ }^{1, \mathrm{~b}}$, Tingting Hua ${ }^{1, \mathrm{~d}}$, \\ ${ }^{1}$ Faculty of Science, Jiangsu University, \\ Zhenjiang 212013, China

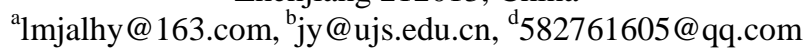

\author{
Yuanyuan $\mathrm{Xu}^{2, \mathrm{c}}$ \\ ${ }^{2}$ School of Mechanical Engineering, \\ Jiangsu University, \\ Zhenjiang 212013, China \\ c723986680@qq.com
}

\author{
Yawei Wang,2,e \\ ${ }^{1}$ Faculty of Science, \\ Jiangsu University, \\ Zhenjiang 212013, China \\ ${ }^{2}$ School of Mechanical Engineering, \\ Jiangsu University, \\ Zhenjiang 212013, China \\ ejszjwyw@sina.cn
}

\begin{abstract}
The studying standard of life sciences and clinical medicine is determined by the development level of morphology detection of biological cells. Currently, there are two approaches for revealing the 3D structure of biological cells, namly, interferometric technique and diffractive technique. Both methods could offer the phase information of the cell. Due to the translucent characteristics of cells and the wave nature of light based on cell scale, interference effect coexists with the diffraction effect when light passes through the cells. In this paper, models based on the shape of the typical blood cells are built and simulation experiments of interference as well as diffraction phase imaging are carried out with the models. Furthermore, the similarities and differences between them are discussed, with respect to the intensity distribution and phase distribution. The result agrees well with the experiments. It is suggested to be useful to promote the research of phase recovery methods of the two kinds of imaging technology.
\end{abstract}

Keywords-Phase microscopy imaging; Biological cells; Interference; Diffraction; Comparative study.

\section{INTRODUCTION}

It is significant to identify the structure of cells in life sciences and clinical applications. Phase microscopy, especially quantitative phase microscopy plays an important role for the structure analysis of biological cells. But the phase information could not be obtained directly because of the limitations of the property of optical devices. Thus, the method of wavefront phase detection has become a hot issue, and wavefront sensing technologies based on interference and diffraction are widely used.

In 1948, Gabor proposed the initial idea of holography [1]. Interferogram of reference light and object light was recorded on the holographic plane, namely, hologram. When hologram is illuminated by reference light, the original object light could be reconstructed based on scalar diffraction theory. In 1967, digital holography [2] has been proposed and electronic device such as a CCD was used to record hologram. The relevant reconstruction could be obtained from simulation by concerning the diffractive process. As for reconstruction algorithm, there are three kinds of algorithms in general [3]: Fresnel transform algorithm, convolution algorithm and angular spectrum algorithm. According to the existence of the angle between object light and reference light on the holographic recording plane, holography can be divided into two types, that is, on-axis holography and off-axis holography. Fourier phase microscopy (FPM) [4] was developed as low-noise phase imaging method. Fourier phase microscopy with white light (wFPM) [5] and Diffraction phase microscopy with white light (wDPM) [6] were developed at the MIT Spectroscopy Laboratory, which have high temporal phase stability and high spatial phase sensitivity. Besides, spatial light interference microscopy (SLIM) [7], slightly off-axis interference [8], Mach-Zehnder interferometer and Michelson interferometer are considered to the main experimental structures of interference microscopy imaging. All of the technologies mentioned above are far-field imaging. E. Cuche et al. [9] and Adam Wax et al. [10] also made outstanding contributions to this field.

Phase inversion technique is active in wavefront detection. The phase information of wavefront could be obtained by this means with different phase retrieval algorithms. In recent years, many phase retrieval algorithms have been developed to improve the accuracy and speed of the technique. In 2004, Campbell proposed phase retrieval algorithm based on multiple diffraction patterns [11]. P.Thibault proposed scanning diffraction imaging technology [12]. These algorithms either reduce the restriction of experimental conditions or simplify the process. But the iterative algorithm could not be avoided. In addition, phase difference detection [13] and multipinhole 
sampling array [14] also can be used to detect wavefront.

According to the above description of imaging techniques for biological cells, we can know that the interferometric and diffractive techniques have their own characteristics and benifits. However, during the process of interferometric quantitative phase imaging, the effects of interference and diffraction exist together due to the wave nature of light. In addition, the diffraction is interference in nature. It is very important to discuss the interaction of the effect of diffraction and interference. We will simulate the interference and diffraction microscopy imaging under the model of transparent sphere in the following. And the characteristics of their intensity distribution as well as phase distribution will be analyzied.

\section{THEORETICAL ANALYSIS OF INTERFERENCE}

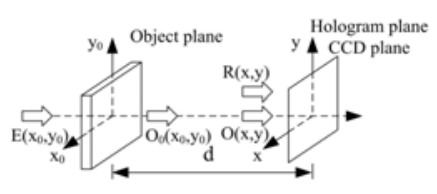

(a)

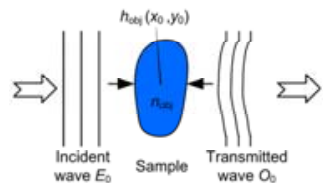

(b)
Fig.1. (a) simplified optical path and the coordinate of interference (b) schematic diagram of light passing through the specimen in the air

As shown in Fig. 1(a), the distance between object plane $x_{0}-y_{0}$ and CCD plane $x-y$ is defined as $d . E\left(x_{0}, y_{0}\right)$ indicates incident light that reaches the object plane, $O_{0}\left(x_{0}, y_{0}\right)$ indicates light that passed through the specimen, which is called as object light for simplification. $O(x, y)$ indicates object light that reaches the CCD plane. $R(x, y)$ indicates reference light on the CCD plane.

The principle of interference imaging could be shown as
Fig. 1(b). The phase is modulated when incident light passes through the specimen in the air. $\varphi_{\text {obj }}\left(x_{0}, y_{0}\right)$ indicates phase delay of the incident light induced by specimen. The relationship between phase delay $\varphi_{o b j}\left(x_{0}, y_{0}\right)$ and optical path difference $\Delta L$ is as follows:

$$
\varphi_{o b j}\left(x_{0}, y_{0}\right)=\frac{2 \pi}{\lambda} \Delta L=\frac{2 \pi}{\lambda}\left(n_{o b j}\left(x_{0}, y_{0}\right)-1\right) h_{o b j}\left(x_{0}, y_{0}\right)
$$

where $n_{o b j}\left(x_{0}, y_{0}\right), h_{o b j}\left(x_{0}, y_{0}\right)$ indicate refraction index and thickness of specimen respectively. If the refractive index of specimen is constant, $\varphi_{o b j}\left(x_{0}, y_{0}\right)$ is proportional to $h_{o b j}\left(x_{0}, y_{0}\right)$, so phase delay of $\mathrm{O}_{0}\left(x_{0}, y_{0}\right)$ reflects the morphology of the specimen. The specimen could be imaged based on the phase distribution of object light. Based on the analysis above, the process of interference imaging method can be summarized as follows: first record an interferogram or a number of interferograms, and then by using different algorithms, extract phase distribution of the object light based on the recorded interferograms, after that reconstruct morphology of specimen. Meanwhile, many approaches have been applied to phase retrieval[15]. For example, the quantitative phase information can be obtained by calculating 1st and 2nd order derivatives of the interferogram in transverse.

\section{THEORETICAL ANALYSIS OF DIFFRACTION}

Generally speaking, the flowchart of wavefront phase detection based on diffraction images is shown as follows.

\begin{tabular}{|c|c|c|c|}
\hline object light & $\begin{array}{l}\text { recording the diffraction } \\
\text { intensity pattern }\end{array}$ & $\rightarrow \begin{array}{c}\text { phase reconstruction } \\
\text { based on algorithm }\end{array}$ & $\rightarrow \begin{array}{c}\text { reconstructing } \\
\text { wavefront }\end{array}$ \\
\hline
\end{tabular}

Fig.2. flowchart of wavefront reconstruction

Iterative algorithm is commonly used in phase inversion technique. Among these methods, input-output algorithm (HIO) and guide hybrid input-output algorithm (GHIO) are actively used. But one of the disadvantages of traditional method is that, it takes long time and demands much about experimental conditions.

As another wavefront detection method, phase difference detection has been proposed. Schematic diagram is shown in Fig. 3.

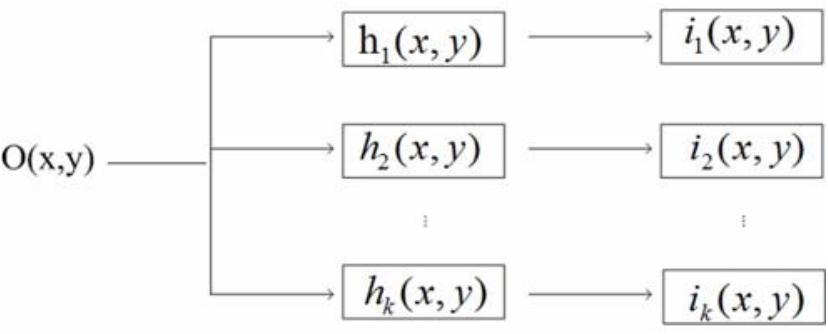

Fig.3. schematic diagram of phase difference detection

$h_{k}(x, y)$ denotes the $\mathrm{k}$ th optical channel, and each optical channel is different from each other. Several images with phase difference are gained after the object light $O(x, y)$ passes through the relevant optical channels. 
Then wavefront phase could be reconstructed by means of iterative algorithm.

Some new wavefront phase detection technologies have been developed in the last decades by using multipinhole sampling array. After object light passes through the multipinhole, the Fraunhofer far-field diffraction intensity pattern is recorded. Then we can reconstruct three-dimensional structure of biological cells based on the recorded diffraction intensity pattern.

\section{COMPARISON OF IMAGE FEATURE BETWEEN}

\section{INTERFERENCE AND DIFFRACTION MICROSCOPY IMAGING}

From the above analysis, it is suggested that structure of biological cells can be reconstructed based on phase information with either interferometric or diffractive theory. We will make a model to investigate the interference effect as well as diffraction effect. In view of the shape of blood cell is sphere, we select a ball as the model. The related parameters are fixed as follows: the diameter is defined at $10 u m$ and the refraction index is fixed at 1.306. Plane-wave laser is used as a source, whose wavelength is $488 \mathrm{~nm}$ with vertical polarization mode. The wrapped phase images of sample based on interference and diffraction microscopy imaging are obtained respectively by simulation.

Fig. 4(a) shows wrapped phase image and distribution curve of the model based on interference method. In order to indicate the effectiveness of this result, wrapped phase image of a polystyrene sphere by simulation as well as the associated experimental wrapped phase image[16] are also demonstrated (shown in Fig. 4(b) and Fig. 4(c)). It should be noted that, the diameter and the refraction index of this polystyrene sphere are set at 40um and 1.5997 respectively. The simulation result agrees well with the optical experiment.

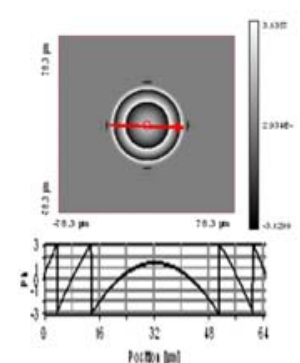

(a)

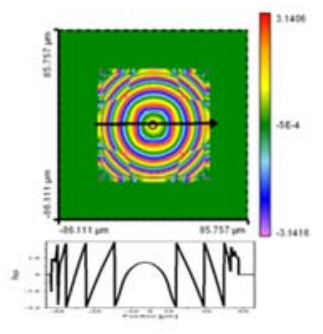

(b)

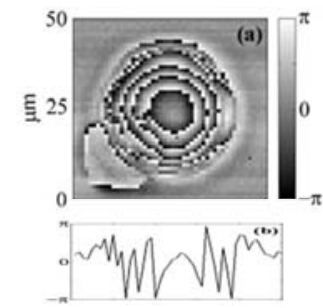

(c)

Fig.4. the phase image based on interference.(a)wrapped phase image and distribution curve of the model.(b)simulated wrapped phase image and distribution curve of polystyrene sphere.(c)experimental wrapped phase image and distribution of polystyrene sphere.

Using the same sphere model (10um) and source with interference microscopy imaging, we simulate diffraction microscopy imaging. Fig. 5(a) shows intensity distribution of diffraction, Fig. 5(b) shows wrapped phase image and Fig. 5(c) shows unwrapped phase image as well.

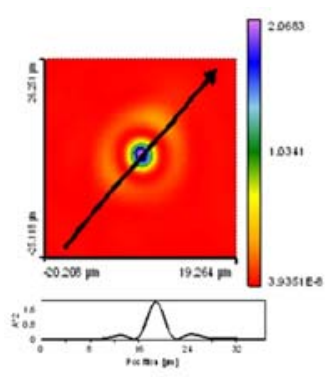

(a)

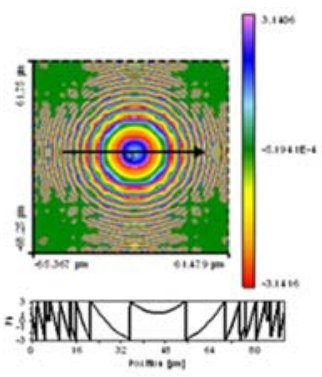

(b)

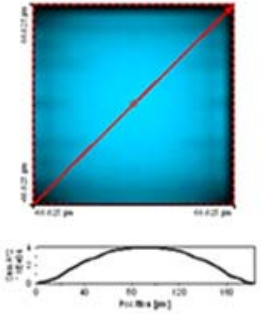

(c)

Fig.5. the image based on diffraction.(a)diffraction intensity distribution.(b)wrapped phase image.(c)unwrapped phase image.

From the phase image shown in Fig. 4 and Fig. 5, phase shift induced by the sample can be observed by interference as well as diffraction. It suggests that both these two methods can be used to reconstruct structure of specimen based on phase information. In the diffractive simulated experiment, the central part of the image has the same shape with the interferometric phase image. It's worth pointing out that, the difference between these two results also could be observed, that is, there are several diffraction stripes surrounding the field of the interferometric phase image in the diffraction image because of the effect of diffraction. Fig. 6 and Fig. 7 are the corresponding result from optical experiment. Fig. 6 shows phase microscopy imaging based on off-axis interference, without reference light. Fig. 7 shows the interferometric micrograph under the same experimental conditions with reference light. In other words, 
the former is just the result of diffraction image while the latter is the result of interference. Comparing Fig. 6 and Fig. 7 , we can find that the size and the shape of the image are the same in the middle of the strip. It agrees well with the results and conclusions of simulation.

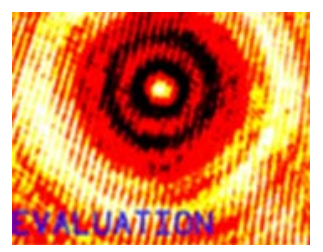

Fig.6. single-beam diffraction pattern

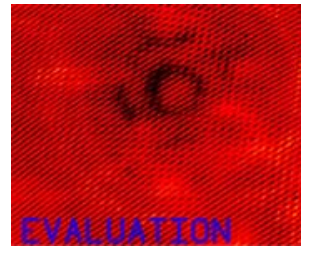

Fig.7. interference micrograph

\section{CONCLUSION}

The morphology of biological cells is very complex, which is not only related to the types of cells, but also related to life stage of it. This complexity will affect the accuracy of calculation and analysis based on either interferometric theory or diffractive theory. Thus, it is difficult to obtain the accurate result. In this paper, we carried out comparative study based on ball model. Simulations of interference phase imaging and diffraction phase imaging are presented. It could be found that phase distribution maps of these two methods have the same characteristics, which is also verified by the optical experiments. This work may have some reference for phase recovery based on interference and diffraction. While the relationship and interaction between these two kinds of phase recovery methods are still the open problems.

\section{ACKNOWLEDGMENTS}

This work was supported by National Natural Science Foundation of China (NO. 11374130, No.11474134), the Doctoral Program Joint Fund of colleges and universities Specialized Research (NO.20113227110018), Post-doctoral Science Fund of China (Grant No 2014M561574) and Innovation Program for College Graduates of Jiangsu Province (NO. KYLX_1017).

\section{REFERENCES}

[1] D. Gabor: Nature Vol. 161 (1948), p. 777.

[2] J.W. Goodman and R.W. Lawrence: Applied Physics Letters Vol. 11 (1967), p. 77

[3] H.Y. Wang, B.Q. Zhao and W. Liao: IEEE International Conference on Intelligent Computing and Intelligent Systems (Comparision of the Reconstruction Algorithms in Digital Micro-holography, Shanghai PEOPLES R CHINA 2009).

[4] G. Popescu, L.P. Deflores and J.C. Vaughan: Opt. Lett. Vol. 29 (2004), p. 2503.

[5] B. Bhaduri, K. Tangella and G. Popescu: Biomed.Opt. Exp. Vol. 4 (2013), p. 1434.

[6] B. Bhaduri, H. Pham, M. Mir and G. Popescu: Opt. Lett. Vol. 37 (2012), p. 1094.

[7] Z. Wang, L. Millet, M. Mir, H.F. Ding, et al.: Opt. Exp. Vol. 19 (2011), p. 1016.

[8] N.T. Shaked, Y.Z. Zhu, M.T. Rinehart and A. Wax: Opt. Exp. Vol. 17 (2009), p. 15585.

[9] P. Marquet, B. Rappaz, P.J. Magistretti, E. Cuche, et al.: Opt. Lett.
Vol. 30 (2005), p. 468.

[10] N.T. Shaked, T.M. Newpher, M.D. Ehlers and A. Wax: Appl. Opt. Vol. 49 (2010), p. 2872.

[11] H.I. Campbell, S.J. Zhang, A.H. Greenaway and S. Restaino: Opt.Lett. Vol. 29 (2004), p. 2707.

[12] P. Thibault, M. Dierolf, A. Menzel, O. Bunk, et al.: Science Vol. 321 (2008), p. 379.

[13] J.J. Dolne, P. Menicucci, D. Miccolis, K. Widen, et al.: Appl.Opt. Vol. 48 (2009), p. A30.

[14] N. Nakajima: Opt.Lett. Vol. 36 (2011), p. 2284.

[15] Y.Y. Xu, Y.W. Wang, W.F. Jin, Y. Ji, et al.: Laser \& Optoelectronics Progress Vol. 51 (2014), p. 020006-1.(In Chinese)

[16] A. Ahn, C. Yang, A. Wax, G. Popescu, et al.: Appl.Opt. Vol. 44 (2005), p.1188. 